\title{
Task Scheduling of a Distributed Computing Software in the Presence of Faults
}

\author{
Kamal Sheel Mishra \\ Department of Computer Engineering \\ Indian Institute of Technology \\ Banaras Hindu University, Varanasi, India
}

\author{
Anil Kumar Tripathi \\ Department of Computer Engineering \\ Indian Institute of Technology \\ Banaras Hindu University, Varanasi, India
}

\begin{abstract}
Performance estimation of a distributed software is a challenging problem. A distributed software runs on multiple processing nodes interconnected in some fashion. In such a situation computational load of a software is distributed onto the processing nodes of the given system. Such a system makes use of an appropriate task scheduling algorithm for obtaining a good performance. The program used in this work emulates a distributed system . An emulator gives the result like an actual system. The emulator is of a fully connected distributed system in which any two processors can directly communicate. The objective of this experiment is to identify the task scheduling algorithm that also performs well in the presence of communication fault delay occured because of network failure or computation fault delay occured because of no response from processors in a distributed system.
\end{abstract}

\section{General Terms:}

Distributed system, Task scheduling

\section{Keywords:}

Clustering, distributed computing, homogeneous systems, scheduling, task allocation.

\section{INTRODUCTION}

Distributed computing software is factually a job that consists of multiple tasks and the performance of the software heavily depends on allocation of these tasks of the DCS (Distributed Computing Software) onto the multiple processing nodes that need to be employed for achieving concurrency for the purpose of ensuring the possible reduction in the turnaround time of a DCS as compared to its corresponding execution on a single processing node in sequential manner.

A DCS can be modeled as a job represented as a task graph showing the execution time of task, communication requirements among the task and some possible precedence constraints and relationships that need to be honored while executing these tasks of a DCS job concurrently. Similarly the multiple processing node distributed computing infrastructure can also be modeled as a processor graph where nodes (vertices) of the graph represent processing nodes along with their attributes showing capabilities, and the links represent the connectivity between some two nodes and communication speed /latency(delay) between the two nodes.

A good performance of a DCS job can be ensured if the tasks are appropriately mapped onto processing nodes of the distributed computing system minimizing inter task communication over- head considering the delays involved between the nodes and ensuring fast computation on the processing nodes.

Such a mapping of task has been studied in literature as a task scheduling problem in distributed computing systems.Many task allocation algorithms have been proposed during the last two decades. This program emulates a distributed system . An emulator gives the result very much similar to an actual system. The emulator is of a fully connected distributed system in which any two processors can directly communicate. Here homogenious nodes have been considered. The main objective of this experiment is to find out the task scheduling algorithm that best performs in the presence of communication fault delay or computation fault delay as well as to identify the algorithm that performs worst in the presence of above faults. For experiment purpose we have taken only six Task Scheduling Algoriths out of many.Further, Communication fault delay may be constant or random. Similarly Computation fault delay may be constant or random. The above faults are evaluated under following three parameters: (i) Normalized schedule length, (ii) Average number of processors used and (iii) Average running time. Using above parameters we identify the algorithms that performs best as well as worst in the presence of faults in the distributed system.

This paper is organised as follows. Section 2 outlines the computation and communication fault delay which may be present in the distributed environments. Section 3 discusses the different task scheduling algorithms used for performance evaluation. In this paper we are considering only six task scheduling algorithms for experiment purpose. In section 4 different performance evaluation parameters used are disscussed. Section 5 shows the related work done in this area. Section 6 shows the experimental setup used in this work. Section 7 shows the performance results for constant fault delays. Section 8 gives the performance results for random fault delay. Section 9 summarizes the performance results as well as the future work to be done. Lastly section 10 gives the list of different references used in writing this paper.

\section{COMPUTATION AND COMMUNICATION FAULT DELAY IN DISTRIBUTED ENVIRONMENTS}

\subsection{Computation fault delay}

Computation fault delay may occur in a distributed system due to hardware failure or machine not responding or a processor is not ready.

\subsection{Communication fault delay}

Communication fault delay may occur because of network failure or congession of network or link failure. 


\subsection{Computation and Communication fault delay}

it is possible that both communication and computation fault delay may occur simultaneously in the system because of hardware or network failure.

\section{TASK SCHEDULING ALGORITHMS USED FOR PERFORMANCE EVALUATION}

We considered six task sheduling algorithms for performance evaluation[1].

1. CPPS algorithm: The Cluster pair priority scheduling algorithm is a cluster dependent function of tasks.

2. DCCL algorithm: The Dynamic computation communication load schedule algorithm is based on a computation and communication time of the module and current allocation.

3. DSC algorithm: The Dominant sequence clustering algorithm is based on the critical path of the graph.

4. EZ algorithm: Edge zeroing algorithm is used to minimize the communication delay. based on edge weight it select clusters for merging []

5. LC algorithm: The linear clustering algorithm is used to create clusters in a parallel system. It merges nodes iteratively to form a single clusster based on critical path.

6. RDCC algorithm: The Randomized computation communication load scheduling algorithm is the dynamic priority version of randomized computation and communication load algorithm.

\section{PERFORMANCE EVALUATION PARAMETERS USED}

1 NSL : Normalized schedule length[1] is the schedule lenght over the sum of computation cost on the critical path of the task graph.

$$
N S L=S L / \sum_{v \in C P} w(v)
$$

where $S L$ is the schedule length and $w(v)$ is the computation cost. 2 Average number of processor used: It is the average of the number of pocessors used in computation of the task graph. 3 Average running time: It is the average of running time used in computing the task in the presence of computation fault, communication fault or both (computation and communication fault) delay.

\section{RELATED WORK}

Alexey lastovetsky[2] focused on using parallel computing technologies to accelerate the testing of a complex distributed software system.Cyril Briquet[3] supported on evaluating the performance of scheduling algorithms. Performance is evaluated experimentally or through simulation. Giovanni Denaro[4] worked on early performance testing of distributed software application.Jmes D herbsleb[5] worked on the extent of delay in a distributed software development organization and explore possible mechanism for this delay. Raul Ceretta Nunes [6] focuses on modeling communication delays in distributed software systems using time series. Yizheng Yao [7] presented a framework for testing distributed software components. Roger Ferguson [8] presented a chaining approach for automated software test data generation for distributed software. Carl K. Chang [9] presented a specification based testing method for distributed software.kwok[10] worked on benchmarking and comparison of the task graph scheduling algorithms. kequin[11] focuses on scheduling parallel tasks on multiprocessor computers with efficient power management. Stankovic[12] worked on evaluation of a flexible task scheduling algorithm for distributed hard real time systems. Tondre[13] presented a technical computation and communication delay in distributed system. Nunes[14] worked on modeling communication delays in distributed systems using time series.

\section{EXPERIMENTAL SETUP}

Evaluate-Time $(T$, cluster $)$

01 time $\leftarrow 0$

02 event $\leftarrow \leftarrow$ empty

03 icount $\leftarrow 0$

04 dcount $\leftarrow 0$

05 for $k \leftarrow 1$ to $|V|$

$06 \quad$ do $\operatorname{status}(k) \leftarrow i d l e$

07 readyq $(k) \leftarrow$ empty

$08 \quad \operatorname{backlink}(k) \leftarrow 0$

09 for $k \leftarrow 1$ to $|V|$

10 do for each $(k, m) \in E$

11 do backlink $(m) \leftarrow \operatorname{backlink}(m)+1$

12 for $k \leftarrow 1$ to $|V|$

13 do if $\operatorname{backlink}(k)=0$

15 for $k \leftarrow 1$ to $|V|$

16 do if $\operatorname{backlink}(k)=0$

17 then if status $($ cluster $(k))=i d l e$

18 then $l \leftarrow \operatorname{DELETE}-\mathrm{QUEUE}($ cluster $(k))$

19 INSERT-HEAP $\left(l, l\right.$, time $\left.+m_{l}\right)$

$20 \quad$ status $($ cluster $(l)) \leftarrow$ busy

$21 \quad$ icount $\leftarrow$ icount +1

22 while true

23 do $(i, j, t) \leftarrow$ Delete-HeAP ()

24 dcount $\leftarrow$ dcount +1

25 time $\leftarrow t$

26 if $($ icount $=(|V|+|E|))$ and $($ dcount $=(|V|+|E|))$

$$
27 \text { then break }
$$

$28 \quad$ if $i=j$

29 then status $($ cluster $(i)) \leftarrow$ idle

30 for each $(i, m) \in E$

$31 \quad$ do if cluster $(i) \neq$ cluster $(m)$

32 then INSERT-HeAP $\left(i, m\right.$, time $\left.+w_{i m}\right)$

33 else INSERT-HEAP $(i, m$, time $)$

$34 \quad$ icount $\leftarrow$ icount +1

$35 \quad l \leftarrow$ Delete-Queue $($ cluster $(i))$

$36 \quad$ if $l \neq$ error

Evaluate-Time (Simulator) calculates the time taken by a given clustering (Mishra et al. [15]). Line 01 initializes time to 0 . Event queue model is used to calculate this time. In this model, we simulate the computations and communications of modules on $n$ machines $W_{k}(1 \leq k \leq n)$. There are two types of events: computation completion event, and communication completion event. Each event is denoted by a 3-tuple $(i, j, t)$. Computation completion event of a module $M_{i}$ is denoted as $(i, i, t)$, where $t$ is the time at which $M_{i}$ finishes its computation. Communication completion event of a communication from a module $M_{i}$ to another module $M_{j}$ is denoted as $(i, j, t)$, where $\mathrm{t}$ is the time at which the communication is completed. As time passes, events are added to, and deleted from the event queue eventq, that is a min-heap based on the value of $t$. In line 02 , we initial- 
ize eventq to empty. icount measures the number of events that are inserted into eventq. dcount measures the number of events that are deleted from eventq. There are a total of $|V|$ events that are computation completeion events corresponding to each module, and a total of $|E|$ events that are communication completion events corresponding to each edge in the task graph. Therefore, a total of $(|V|+|E|)$ events are inserted to, and deleted from eventq. In lines 03 and 04, icount and dcount are initialized to 0 .

status : $V \rightarrow\{$ idle, busy $\}$ is a function that represents the status of the machines $W_{k}(1 \leq k \leq n)$. status $(k)=i d l e$, if and only if the machine $W_{k}$ is not executing any module. $\operatorname{status}(k)=$ busy, if and only if the machine $W_{k}$ is executing a module that is allocated to it. In line 06, status of each machine is initialized to idle.

Each machine $W_{k}(1 \leq k \leq n)$ has a queue of modules readyq $(k)$ associated with it, that are ready to run. A module is ready to run, if it has completed all of its communication completion events. In line 07, readyq is initialized to empty for each machine.

backlink : $V \rightarrow \mathcal{Z}^{+}$is a function that counts the number of waiting communication completion events for each module. In line 08 , backlink is initialized to 0 for each module. In lines 09 to 11 , backlink is initialized to the number of incoming edges for each module. A module is ready to run, if its backlink value is 0 .

In lines 12 to 14 , all ready to run modules are inserted into readyq of the machine to which they are allocated, using the function INSERT-QUEUE. In lines 15 to 21, for all machines that are having ready to run modules, their first ready to run module is deleted from readyq, using the function DELETE-QueUE. Then its computation completion event is inserted into eventq, using the function INSERT-HEAP. For getting the computation completion time, we simply add the execution time of the module to the current time. status of the machine is set to busy, indicating that it is executing a module. icount is updated to count the number of events inserted into eventq.

The while loop from lines 22 to 48 is repeated until all the $(|V|+|E|)$ events are added to, and deleted from readyq (lines 26 to 27 ). In lines 23 to 25 , the first event on eventq is deleted using Delete-HEAP. dcount is updated to count the number of events deleted from eventq. time is set to the completion time of the event. Now we have two possibilities for the deleted event. It can be a computation completion event, or a communication completion event.

Lines 28 to 39 handle the case of a computation completion event. In line 29, the status of the machine on which the module was executing, is set to idle. In lines 30 to 34 , the communication completion events corresponding to each outgoing edge from the module are added to eventq, and icount is updated. The event completion time is set to the current time, if the two modules along the edge are allocated to the same cluster (line 33 ). If the modules belong to different clusters, then the event completion time is set to the current time added with the edge weight (line 32 ). In lines 35 to 39 , another ready to run module (if one exists) is deleted from the readyq of the machine. Its computation completion event is added to eventq, icount is updated, and the status of the machine is set to busy.

Lines 40 to 48 handle the case of a communication completion event. In line 40 , the backlink of the destination module (of communication) is decremented. In lines 41 to 42 , if the backlink value is zero, then this module has become ready to run, and is added to the readyq of the machine to which it is allocated. In lines 43 to 48 , if this machine is idle, then the first ready to run module (if one exists) is deleted from its readyq. Its computation completion event is added to eventq, icount is updated, and the status of the machine is set to busy. The final value of time is returned in line 49 .
Lines 01 to 04 has complexity $O(1)$. Lines 05 to 08 has complexity $O(|V|)$. Lines 09 to 11 has complexity $O(|V|+$ $|E|)$. INSERT-QUEUE has complexity $O(1)$. Therefore, lines 12 to 14 has complexity $O(|V|)$. Delete-Queue has complexity $O(1)$. INSERT-HEAP and DELETE-HEAP have complexity $O(\log (|V|+|E|))$ (Cormen et al. [16], Horowitz and Sahni [17], Langsam et al. [18]). Therefore, lines 15 to 21 has complexity $O(|V| \log (|V|+|E|))$. In the while loop from lines 22 to 48 , there are $|V|$ computation completion events, all inserting a total of $|E|$ communication completion events into eventq, giving a complexity of $O(|E| \log (|V|+|E|))$. There are $|E|$ communication completion events, all inserting a total of $|V|$ computation completion events into eventq, giving a complexity of $O(|V| \log (|V|+|E|))$. Also a total of $(|V|+|E|)$ events are deleted from eventq, giving a complexity of $O((|V|+$ $|E|) \log (|V|+|E|))$. Line 49 has complexity $O(1)$. Therefore, EVALUATE-TIME has complexity $O((|V|+|E|) \log (|V|+|E|))$.

\section{PERFORMANCE RESULTS FOR CONSTANT FAULT DELAY}

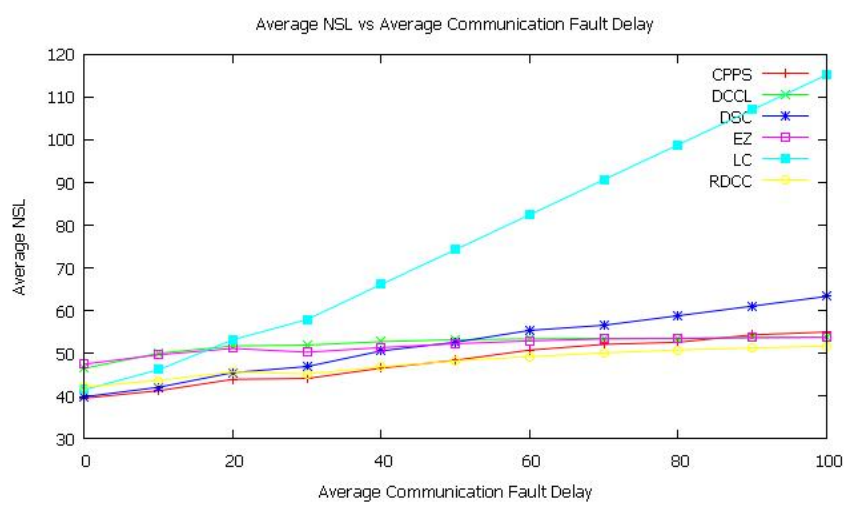

Fig. 1. Average NSL vs average communication fault delay for constant communication fault delay. The average percentage variation order of NSL is:

$E Z<D C C L<R D C C<C P P S<D S C<L C$.

Figure 1 shows the average NSL vs average communication fault delay for constant communication fault delay. Average percentage variation of NSL for CPPS ranges from 0.000000 to 39.339941 with an average of 21.689623 . Average percentage variation of NSL for DCCL ranges from

0.000000 to 15.701477 with an average of 12.238085 . Average percentage variation of NSL for DSC ranges from 0.000000 to 59.248132 with an average of 30.824493 . Average percentage variation of NSL for EZ ranges from 0.000000 to 13.166381 with an average of 9.015570 . Average percentage variation of NSL for LC ranges from 0.000000 to 178.140955 with an average of 82.813149 . Average percentage variation of NSL for RDCC ranges from 0.000000 to 22.822389 with an average of 13.321739. The average percentage variation order of NSL is: $E Z<D C C L<R D C C<C P P S<D S C<L C$.

Figure 2 shows the average number of processors used vs average communication fault delay for constant communication fault delay. Average percentage variation of number of processors used by CPPS ranges from -77.336198 to 0.000000 with an average of -53.925398 . Average percentage variation of number of processors used by DCCL ranges from -58.125000 to 0.000000 with an average of -42.613636 . Average percentage variation of number of processors used by DSC ranges from 57.110609 to 0.000000 with an average of -37.081880 . Average 


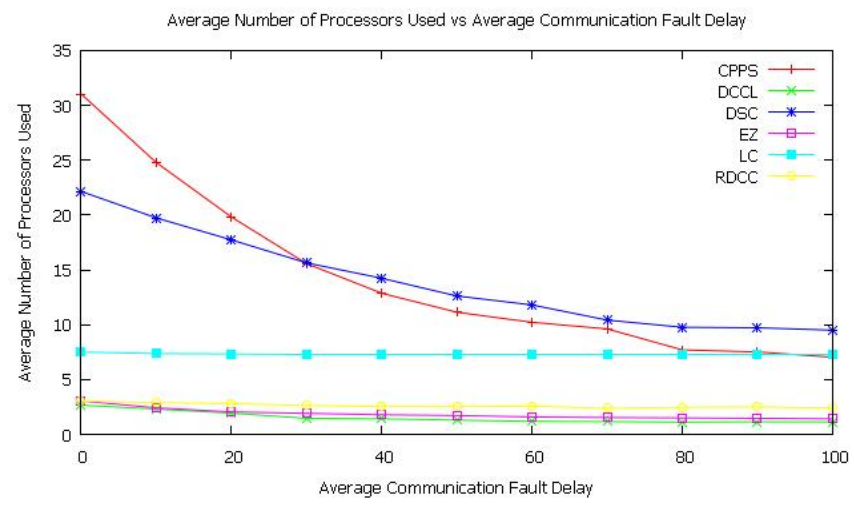

Fig. 2. Average number of processors used vs average communication fault delay for constant communication fault delay.

The average percentage variation order of average number of processors used is:

$C P P S<D C C L<E Z<D S C<R D C C<L C$.

percentage variation of number of processors used by EZ ranges from -53.551913 to 0.000000 with an average of -39.095877 . Average percentage variation of number of processors used by LC ranges from -2.888889 to 0.000000 with an average of 2.181818. Average percentage variation of number of processors used by RDCC ranges from -22.404372 to 0.000000 with an average of -14.356682 . The average percentage variation order of average number of processors used is: $C P P S<D C C L<$ $E Z<D S C<R D C C<L C$.

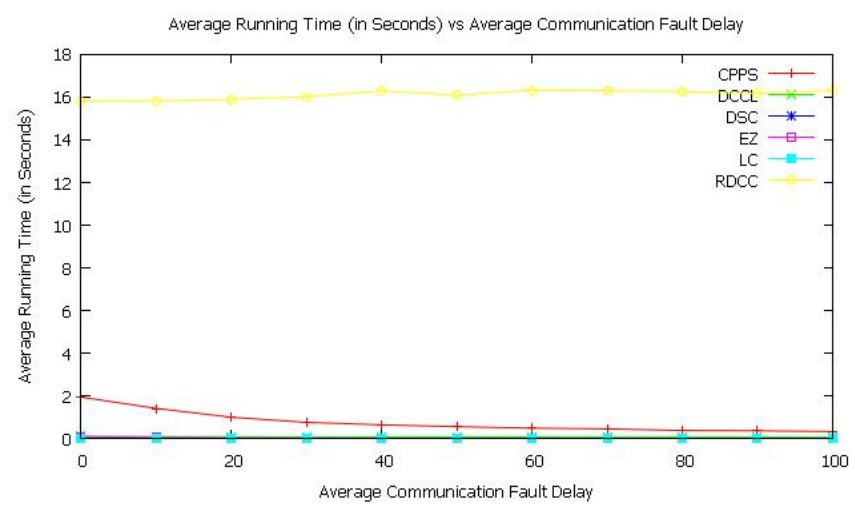

Fig. 3. Average running time (in seconds) vs average communication fault delay for constant communication fault delay. The average percentage variation order of average running time (in seconds) is: $E Z<C P P S<D C C L<R D C C<D S C<L C$.

Figure 3 shows the average running time (in seconds) vs average communication fault delay for constant communication fault delay. Average percentage variation of execution time for CPPS ranges from -83.545980 seconds to 0.000000 seconds with an average of -61.296417 seconds. Average percentage variation of execution time for DCCL ranges from -48.230505 seconds to 0.000000 seconds with an average of -32.104530 seconds. Average percentage variation of execution time for DSC ranges from -7.494572 seconds to 23.195832 seconds with an average of 7.153515 seconds. Average percentage variation of execution time for EZ ranges from -84.616829 seconds to $0.000000 \mathrm{sec}-$ onds with an average of -69.172067 seconds. Average percentage variation of execution time for $\mathrm{LC}$ ranges from 0.000000 seconds to 104.359697 seconds with an average of 45.954169 seconds. Average percentage variation of execution time for RDCC ranges from 0.000000 seconds to 3.283782 seconds with an average of 1.987271 seconds. The average percentage variation order of average running time (in seconds) is: $E Z<C P P S<D C C L<$ $R D C C<D S C<L C$.

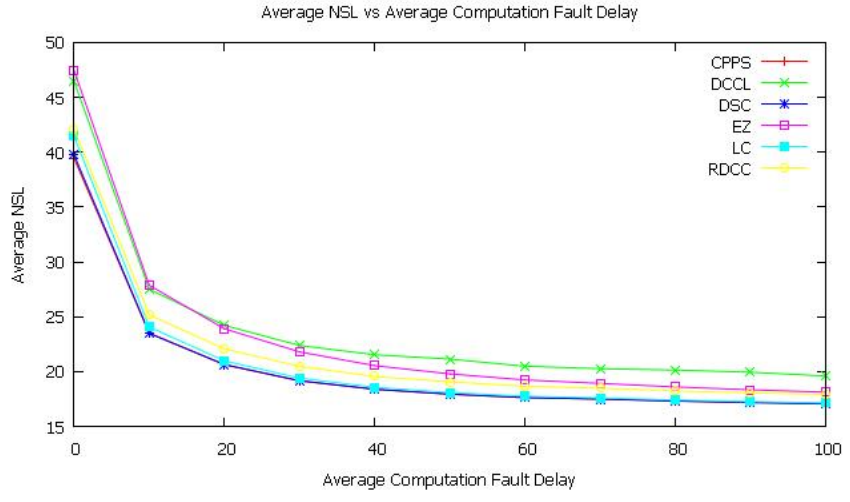

Fig. 4. Average NSL vs average computation fault delay for constant computation fault delay. The average percentage variation order of average NSL is:

$E Z<L C<D C C L<D S C<R D C C<C P P S$.

Figure 4 shows the average NSL vs average computation fault delay for constant computation fault delay. Average percentage variation of NSL for CPPS ranges from -56.728965 to 0.000000 with an average of -47.964772 . Average percentage variation of NSL for DCCL ranges from -57.803176 to 0.000000 with an average of -48.398015 . Average percentage variation of NSL for DSC ranges from -57.064704 to 0.000000 with an average of 48.295160. Average percentage variation of NSL for EZ ranges from -61.778948 to 0.000000 with an average of -51.208754 . Average percentage variation of NSL for LC ranges from 58.625609 to 0.000000 with an average of -49.596531 . Average percentage variation of NSL for RDCC ranges from -57.411905 to 0.000000 with an average of -48.201589 . The average percentage variation order of average NSL is: $E Z<L C<D C C L<$ $D S C<R D C C<C P P S$.

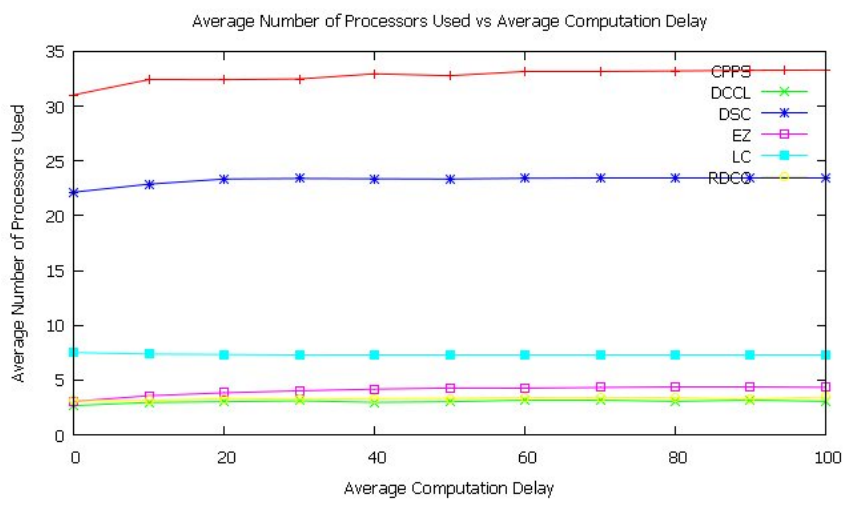

Fig. 5. Average number of processors used vs average computation fault delay for constant computation fault delay. The average percentage variation order of average number of processors used is: $L C<D S C<C P P S<R D C C<D C C L<E Z$. 
Figure 5 shows the average number of processors used vs average computation fault delay for constant computation fault delay. Average percentage variation of number of processors used by CPPS ranges from 0.000000 to 7.303974 with an average of 5.502392. Average percentage variation of number of processors used by DCCL ranges from 0.000000 to 18.750000 with an average of 13.238636. Average percentage variation of number of processors used by DSC ranges from 0.000000 to 5.944319 with an average of 4.979821. Average percentage variation of number of processors used by EZ ranges from 0.000000 to 42.622951 with an average of 32.637854. Average percentage variation of number of processors used by LC ranges from -2.888889 to 0.000000 with an average of -2.181818 . Average percentage variation of number of processors used by RDCC ranges from 0.000000 to 11.827957 with an average of 6.647116 . The average percentage variation order of average number of processors used is: $L C<D S C<C P P S<R D C C<D C C L<E Z$.

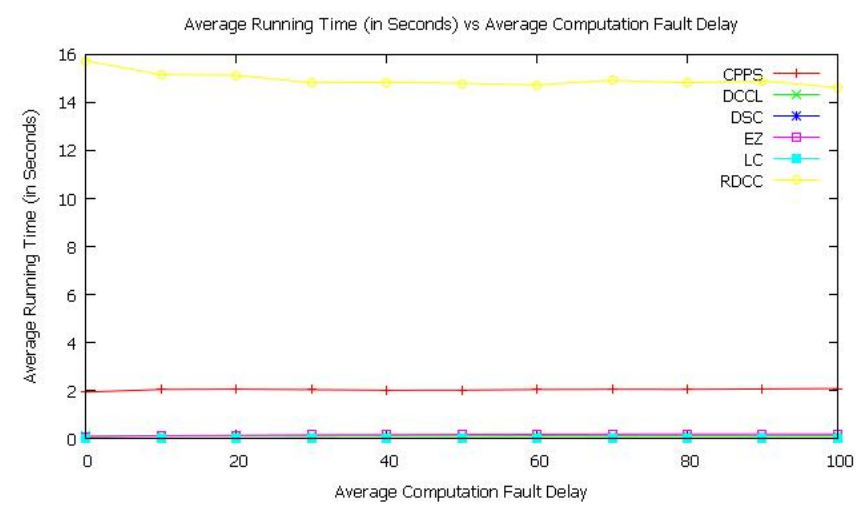

Fig. 6. Average running time (in seconds) vs average computation fault delay for constant computation fault delay. The average percentage variation order of running time (in seconds) is: $R D C C<C P P S<D C C L<D S C<L C<E Z$.

Figure 6 shows the average running time (in seconds) vs average computation fault delay for constant computation fault delay. Average percentage variation of execution time for CPPS ranges from 0.000000 seconds to 7.643489 seconds with an average of 5.144307 seconds. Average percentage variation of execution time for DCCL ranges from 0.000000 seconds to 9.347126 seconds with an average of 5.281630 seconds. Average percentage variation of execution time for DSC ranges from - 11.770253 seconds to 31.426996 seconds with an average of 11.020723 seconds. Average percentage variation of execution time for EZ ranges from 0.000000 seconds to 138.690877 seconds with an average of 99.486318 seconds. Average percentage variation of execution time for LC ranges from -18.176091 seconds to 83.926603 seconds with an average of 25.908160 seconds. Average percentage variation of execution time for RDCC ranges from -7.079550 seconds to 0.000000 seconds with an average of -5.005273 seconds. The average percentage variation order of running time (in seconds) is: $R D C C<C P P S<D C C L<$ $D S C<L C<E Z$.

Figure 7 shows the average NSL vs average computation communication fault delay for constant computation communication fault delay. Average percentage variation of NSL for CPPS ranges from -47.284423 to 0.000000 with an average of 40.515767. Average percentage variation of NSL for DCCL ranges from -46.723321 to 0.000000 with an average of 39.927285. Average percentage variation of NSL for DSC ranges from -48.131674 to 0.000000 with an average of -41.121503 .

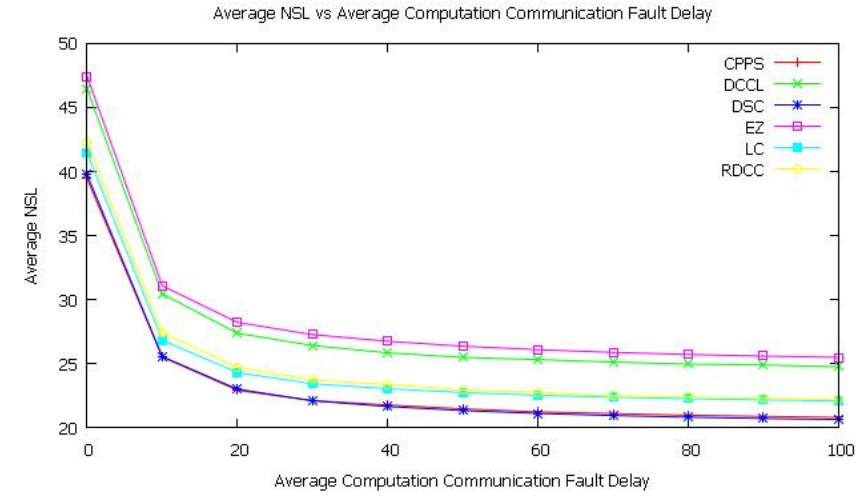

Fig. 7. Average NSL vs average computation communication fault delay for constant computation communication fault delay. The average percentage variation order of NSL is:

$D S C<R D C C<C P P S<L C<D C C L<E Z$.

Average percentage variation of NSL for EZ ranges from 46.297128 to 0.000000 with an average of -39.482014 . Average percentage variation of NSL for LC ranges from -46.719418 to 0.000000 with an average of -40.091101 . Average percentage variation of NSL for RDCC ranges from -47.565465 to 0.000000 with an average of -40.565215 . The average percentage variation order of NSL is: $D S C<R D C C<C P P S<L C<$ $D C C L<E Z$.

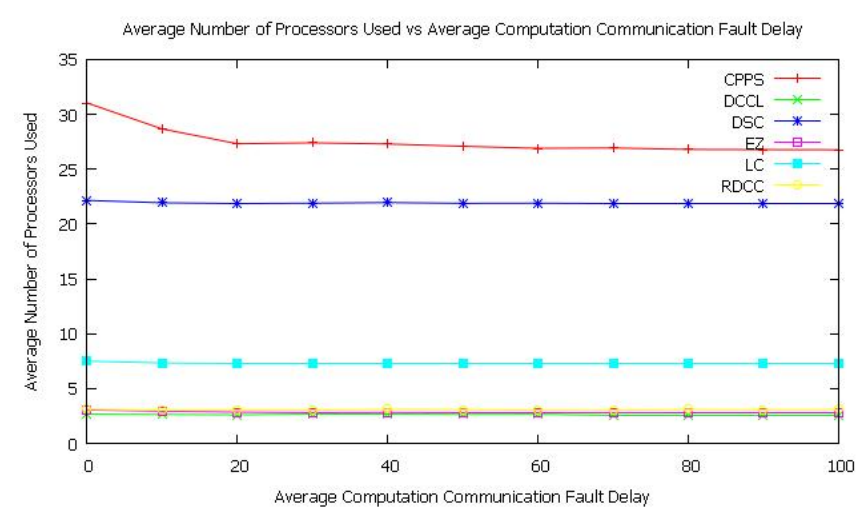

Fig. 8. Average number of processors used vs average computation communication fault delay for constant computation

communication fault delay. The average percentage variation order of number of processors used is:

$C P P S<E Z<L C<D C C L<R D C C<D S C$.

Figure 8 shows the average number of processors used vs average computation communication fault delay for constant computation communication fault delay. Average percentage variation of number of processors used by CPPS ranges from -13.748657 to 0.000000 with an average of -11.244019 . Average percentage variation of number of processors used by DCCL ranges from -3.125000 to 0.000000 with an average of -1.875000 . Average percentage variation of number of processors used by DSC ranges from -1.279157 to 0.000000 with an average of 0.985019 . Average percentage variation of number of processors used by EZ ranges from -8.743169 to 0.000000 with an average of -6.905117. Average percentage variation of number of processors used by LC ranges from -2.444444 to 0.000000 with an average of -2.202020 . Average percentage variation of number of 
processors used by RDCC ranges from -3.743316 to 0.000000 with an average of -1.798736 . The average percentage variation order of number of processors used is: $C P P S<E Z<L C<$ $D C C L<R D C C<D S C$

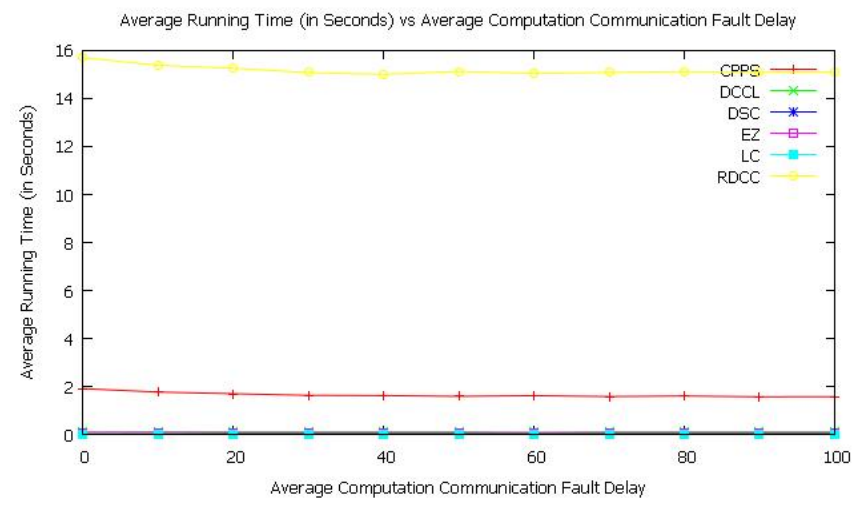

Fig. 9. Average running time (in seconds) vs average computation communication fault delay for constant computation communication fault delay. The average percentage variation order of running time (in seconds) is:

$E Z<C P P S<D C C L<R D C C<L C<D S C$.

Figure 9 shows the average running time (in seconds) vs average computation communication fault delay for constant computation communication fault delay. Average percentage variation of execution time for CPPS ranges from -17.768162 seconds to 0.000000 seconds with an average of -13.567021 seconds. Average percentage variation of execution time for DCCL ranges from -8.409209 seconds to 0.000000 seconds with an average of -6.374468 seconds. Average percentage variation of execution time for DSC ranges from 0.000000 seconds to $45.693766 \mathrm{sec}-$ onds with an average of 23.202806 seconds. Average percentage variation of execution time for EZ ranges from -22.443343 seconds to 0.000000 seconds with an average of $-17.522852 \mathrm{sec}-$ onds. Average percentage variation of execution time for LC ranges from -5.702218 seconds to 53.187698 seconds with an average of 16.200082 seconds. Average percentage variation of execution time for RDCC ranges from -4.436490 seconds to 0.000000 seconds with an average of -3.309152 seconds. The average percentage variation order of running time (in seconds) is: $E Z<C P P S<D C C L<R D C C<L C<D S C$.

\section{PERFORMANCE RESULTS FOR RANDOM FAULT DELAY}

Figure 10 shows the average NSL vs average communication fault delay for random communication fault delay. Average percentage variation of NSL for CPPS ranges from -57.657146 to 0.000000 with an average of -47.261227 . Average percentage variation of NSL for DCCL ranges from -58.716447 to 0.000000 with an average of -47.870314 . Average percentage variation of NSL for DSC ranges from -57.993719 to 0.000000 with an average of -47.624933 . Average percentage variation of NSL for EZ ranges from -62.491137 to 0.000000 with an average of 50.513271. Average percentage variation of NSL for LC ranges from -59.573872 to 0.000000 with an average of -48.991292 . Average percentage variation of NSL for RDCC ranges from -58.371457 to 0.000000 with an average of -47.470166 . The average percentage variation order of NSL is: $E Z<L C<$ $D C C L<D S C<R D C C<C P P S$.

Figure 11 shows the average number of processors used vs average communication fault delay for random communication fault

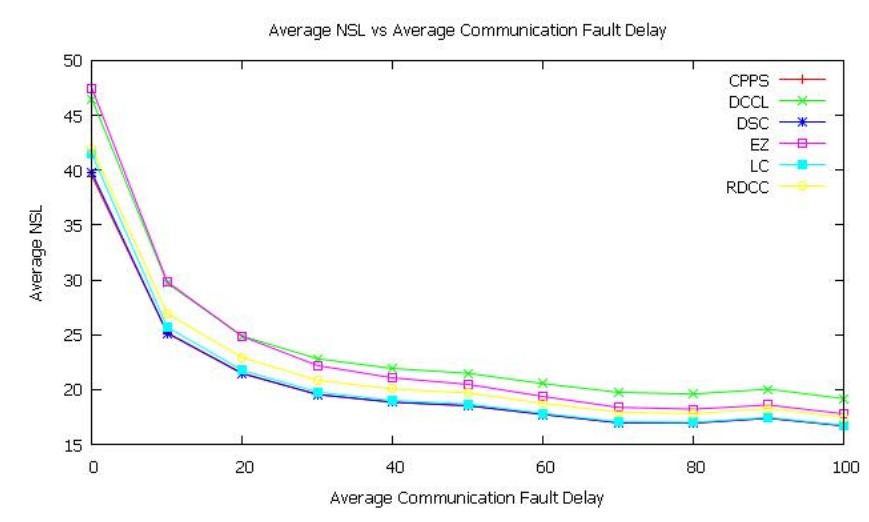

Fig. 10. Average NSL vs average communication fault delay for random communication fault delay. The average percentage variation order of NSL is:

$E Z<L C<D C C L<D S C<R D C C<C P P S$.

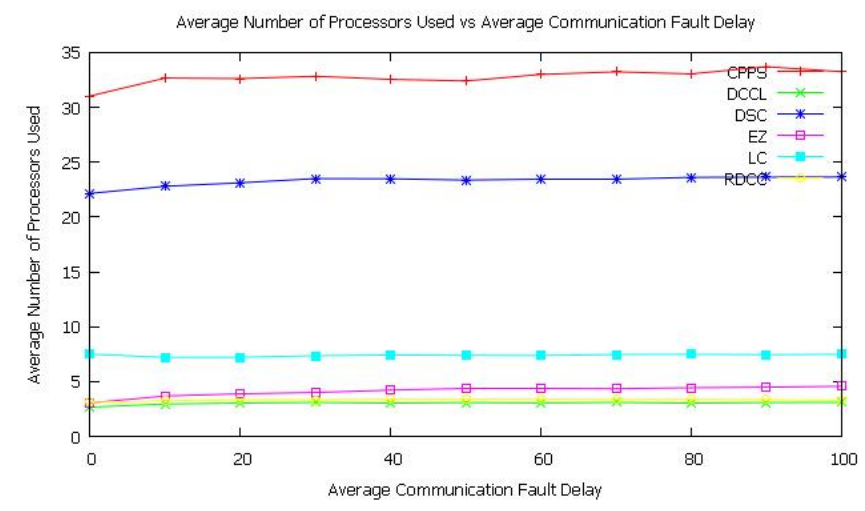

Fig. 11. Average number of processors used vs average communication fault delay for random communication fault delay. The average percentage variation order of number of processors used is: $L C<D S C<C P P S<R D C C<D C C L<E Z$.

delay. Average percentage variation of number of processors used by CPPS ranges from 0.000000 to 8.592911 with an average of 5.556098. Average percentage variation of number of processors used by DCCL ranges from 0.000000 to 16.250000 with an average of 13.295455. Average percentage variation of number of processors used by DSC ranges from 0.000000 to 6.772009 with an average of 5.171352. Average percentage variation of number of processors used by EZ ranges from 0.000000 to 49.726776 with an average of 35.022355 . Average percentage variation of number of processors used by LC ranges from -4.000000 to 0.000000 with an average of -1.434343 . Average percentage variation of number of processors used by RDCC ranges from 0.000000 to 10.752688 with an average of 7.429130.The average percentage variation order of number of processors used is: $L C<D S C<C P P S<R D C C<$ $D C C L<E Z$.

Figure 12 shows the average running time (in seconds) vs average communication fault delay for random communication fault delay. Average percentage variation of execution time for CPPS ranges from 0.000000 seconds to 13.424358 seconds with an average of 9.490876 seconds. Average percentage variation of execution time for DCCL ranges from 0.000000 seconds to 6.794227 seconds with an average of 5.014444 seconds. Average percentage variation of execution time for DSC ranges from 


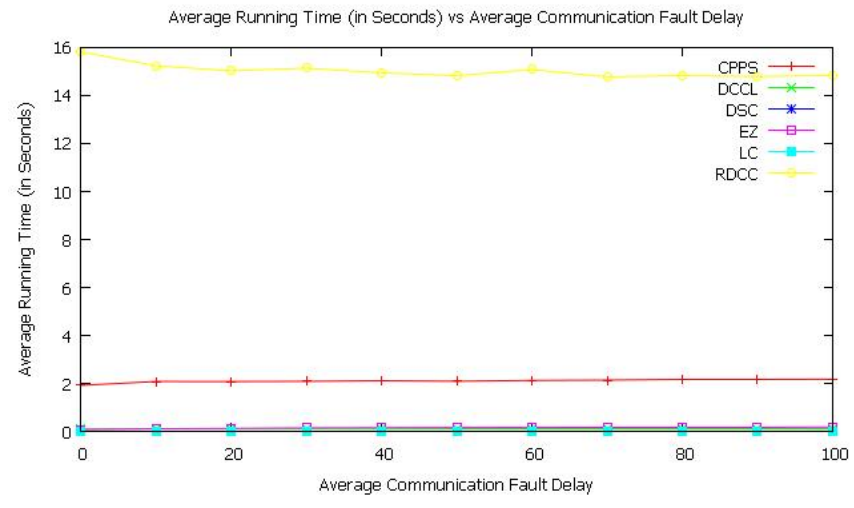

Fig. 12. Average running time (in seconds) vs average communication fault delay for random communication fault delay.

The average percentage variation order of running time (in seconds) is: $R D C C<D C C L<C P P S<D S C<L C<E Z$.

-1.464375 seconds to 58.513965 seconds with an average of 27.081776 seconds. Average percentage variation of execution time for EZ ranges from 0.000000 seconds to $141.977298 \mathrm{sec}-$ onds with an average of 100.551805 seconds. Average percentage variation of execution time for LC ranges from -1.270763 seconds to 137.433058 seconds with an average of 44.802660 seconds. Average percentage variation of execution time for RDCC ranges from -6.555553 seconds to 0.000000 seconds with an average of -4.966295 seconds. The average percentage variation order of running time (in seconds) is: $R D C C<D C C L<$ $C P P S<D S C<L C<E Z$.

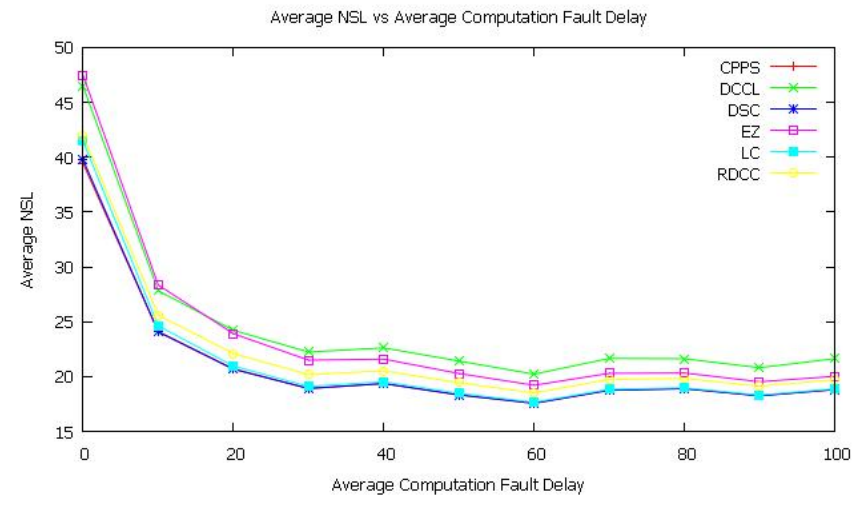

Fig. 13. Average NSL vs average computation fault delay for random computation fault delay. The average percentage variation order of NSL is:

$E Z<L C<D C C L<D S C<R D C C<C P P S$.

Figure 13 shows the average NSL vs average computation fault delay for random computation fault delay. Average percentage variation of NSL for CPPS ranges from -55.454729 to 0.000000 with an average of -46.252097 . Average percentage variation of NSL for DCCL ranges from -56.456555 to 0.000000 with an average of -47.014353 . Average percentage variation of NSL for DSC ranges from -55.813059 to 0.000000 with an average of 46.628080. Average percentage variation of NSL for EZ ranges from -59.497232 to 0.000000 with an average of -49.695130 . Average percentage variation of NSL for LC ranges from 57.369522 to 0.000000 with an average of -48.036695 . Average percentage variation of NSL for RDCC ranges from -55.916115 to 0.000000 with an average of -46.586764 . The average percentage variation order of NSL is: $E Z<L C<D C C L<$ $D S C<R D C C<C P P S$.

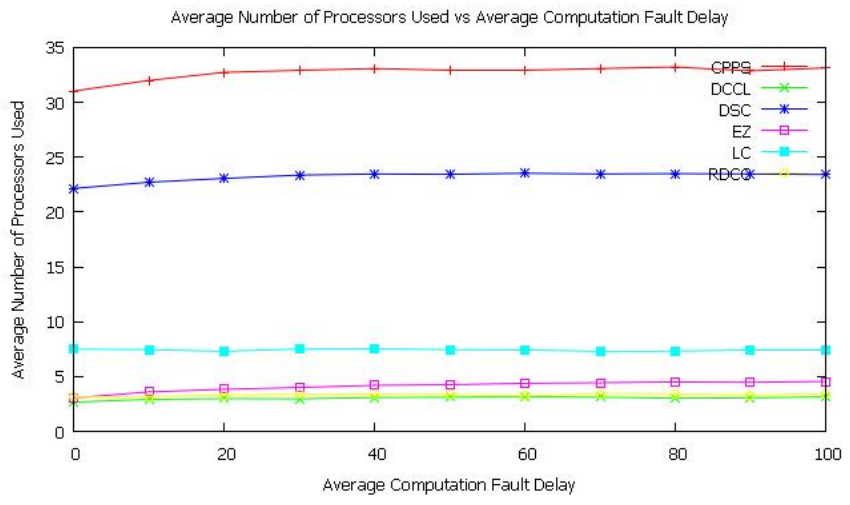

Fig. 14. Average numbeer of processors used vs average computation fault delay for random computation fault delay. The average percentage variation order of number of processors used is: $L C<D S C<C P P S<R D C C<D C C L<E Z$.

Figure 14 shows the average numbeer of processors used vs average computation fault delay for random computation fault delay. Average percentage variation of number of processors used by CPPS ranges from 0.000000 to 7.035446 with an average of 5.438922. Average percentage variation of number of processors used by DCCL ranges from 0.000000 to 18.750000 with an average of 13.295455. Average percentage variation of number of processors used by DSC ranges from 0.000000 to 6.245297 with an average of 4.897736 . Average percentage variation of number of processors used by EZ ranges from 0.000000 to 49.180328 with an average of 34.923000. Average percentage variation of number of processors used by LC ranges from -2.666667 to 0.444444 with an average of -1.070707 . Average percentage variation of number of processors used by RDCC ranges from 0.000000 to 10.695187 with an average of 6.757414 . The average percentage variation order of number of processors used is: $L C<D S C<C P P S<R D C C<D C C L<E Z$.

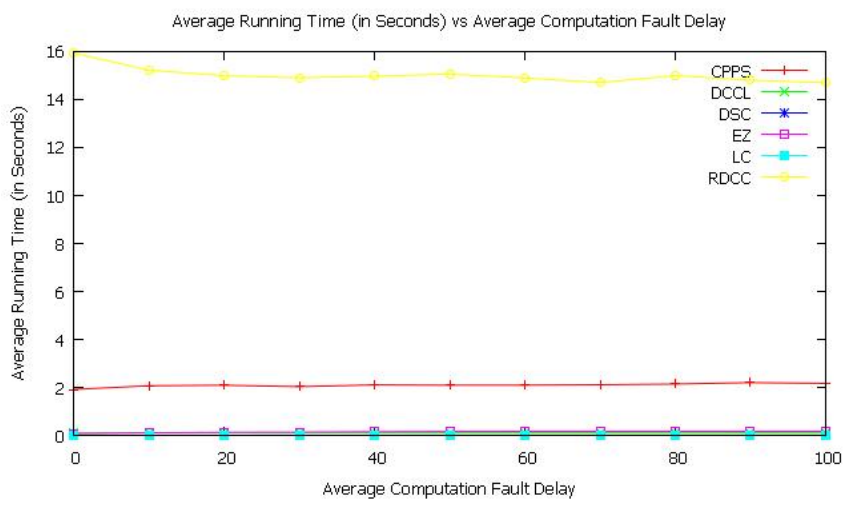

Fig. 15. Average running time (in seconds) vs average computation fault delay for random computation fault delay. The average percentage variation order of running time (in seconds) is: $R D C C<D S C<L C<D C C L<C P P S<E Z$.

Figure 15 shows the average running time (in seconds) vs average computation fault delay for random computation fault delay. 
Average percentage variation of execution time for CPPS ranges from 0.000000 seconds to 14.814304 seconds with an average of 9.175700 seconds. Average percentage variation of execution time for DCCL ranges from 0.000000 seconds to $8.216433 \mathrm{sec}-$ onds with an average of 5.047240 seconds. Average percentage variation of execution time for DSC ranges from -21.159173 seconds to 22.797506 seconds with an average of $-2.713131 \mathrm{sec}-$ onds. Average percentage variation of execution time for EZ ranges from 0.000000 seconds to 139.845090 seconds with an average of 98.640647 seconds. Average percentage variation of execution time for LC ranges from -38.532273 seconds to 49.750831 seconds with an average of 0.479462 seconds. Average percentage variation of execution time for RDCC ranges from -7.767776 seconds to 0.000000 seconds with an average of -5.753271 seconds. The average percentage variation order of running time (in seconds) is: $R D C C<D S C<L C<$ $D C C L<C P P S<E Z$.

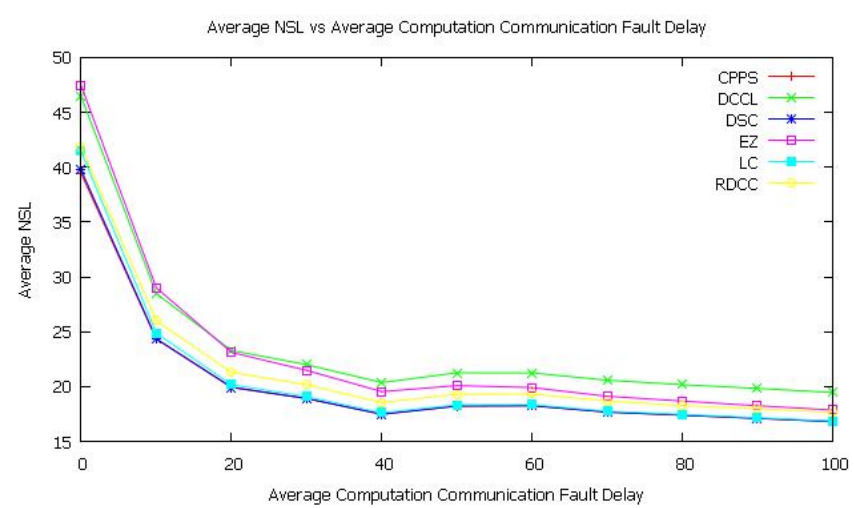

Fig. 16. Average NSL vs average computation communication fault delay for random computation communication fault delay. The average percentage variation order of NSL is: $E Z<L C<D C C L<D S C<R D C C<C P P S$.

Figure 16 shows the average NSL vs average computation communication fault delay for random computation communication fault delay. Average percentage variation of NSL for CPPS ranges from -57.344482 to 0.000000 with an average of -47.993664 . Average percentage variation of NSL for DCCL ranges from -58.097523 to 0.000000 with an average of 48.496198. Average percentage variation of NSL for DSC ranges from -57.701895 to 0.000000 with an average of -48.339627 . Average percentage variation of NSL for EZ ranges from 62.333172 to 0.000000 with an average of -51.220358 . Average percentage variation of NSL for LC ranges from -59.291658 to 0.000000 with an average of -49.722045 . Average percentage variation of NSL for RDCC ranges from -57.842815 to 0.000000 with an average of -48.072150 . The average percentage variation order of NSL is: $E Z<L C<D C C L<D S C<R D C C<$ $C P P S$.

Figure 17 shows the average number of processors used vs average computation communication fault delay for random computation communication fault delay. Average percentage variation of number of processors used by CPPS ranges from 0.000000 to 7.089151 with an average of 5.385216. Average percentage variation of number of processors used by DCCL ranges from 0.000000 to 18.125000 with an average of 12.670455 . Average percentage variation of number of processors used by DSC ranges from 0.000000 to 7.072987 with an average of 5.260278. Average percentage variation of number of processors used by EZ ranges from 0.000000 to 46.448087 with an average of

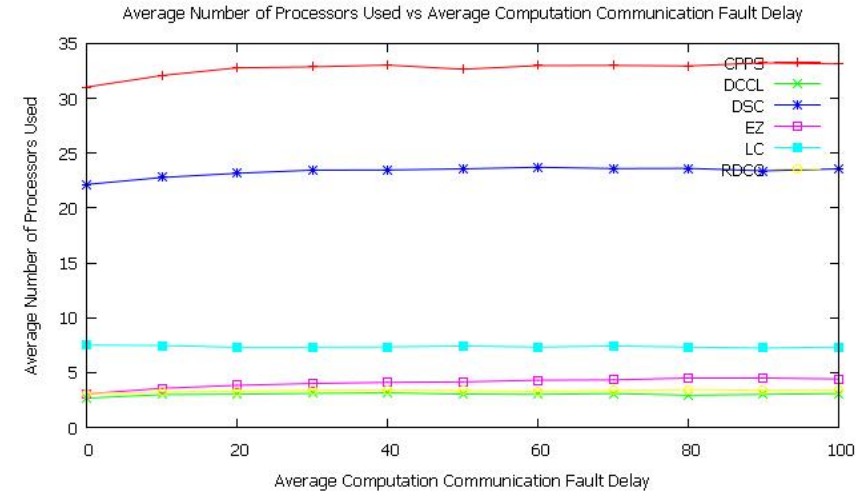

Fig. 17. Average number of processors used vs average computation communication fault delay for random computation communication fault delay. The average percentage variation order of number of processors used is:

$L C<D S C<C P P S<R D C C<D C C L<E Z$.

33.184302. Average percentage variation of number of processors used by LC ranges from -3.555556 to 0.000000 with an average of -1.878788 . Average percentage variation of number of processors used by RDCC ranges from 0.000000 to 11.891892 with an average of 8.157248 . The average percentage variation order of number of processors used is: $L C<D S C<$ $C P P S<R D C C<D C C L<E Z$.

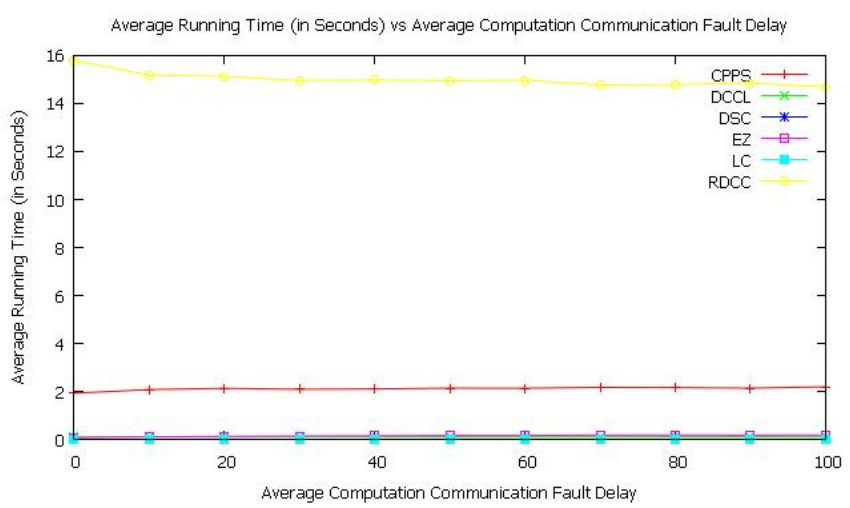

Fig. 18. Average running time (in seconds) vs average computation communication fault delay for random computation

communication fault delay. The average percentage variation order of running time (in seconds) is:

$R D C C<D C C L<C P P S<D S C<L C<E Z$.

Figure 18 shows the average running time (in seconds) vs average computation communication fault delay for random computation communication fault delay. Average percentage variation of execution time for CPPS ranges from 0.000000 seconds to 13.820314 seconds with an average of 9.776371 seconds. Average percentage variation of execution time for DCCL ranges from 0.000000 seconds to 7.923574 seconds with an average of 5.369076 seconds. Average percentage variation of execution time for DSC ranges from 0.000000 seconds to $45.135699 \mathrm{sec}-$ onds with an average of 15.460150 seconds. Average percentage variation of execution time for EZ ranges from 0.000000 seconds to 138.208573 seconds with an average of 97.333918 seconds. Average percentage variation of execution time for LC ranges from -18.562208 seconds to 75.688107 seconds with an 
average of 17.828406 seconds. Average percentage variation of execution time for RDCC ranges from -7.048315 seconds to 0.000000 seconds with an average of -5.005104 seconds. The average percentage variation order of running time (in seconds) is: $R D C C<D C C L<C P P S<D S C<L C<E Z$.

\section{CONCLUSION}

Experiments mentioned in this paper were performed to identify the task scheduling algorithms that also performs well in the presence of communication fault delay or computation fault delay. Six algorithms (CPPS, DCCL, DSC, EZ, LC, RDCC) were evaluated for two types of task graphs (i) task graphs with random fault delay and (ii) task graphs with constant fault delay. From the above graphs and results it can be concluded that for a constant communication delay EZ algorithm gives best result and LC algorithm as the worst result.This may be because EZ algorithm gives non linear clustering and LC algorithm gives linear clustering. RDCC algorithm performs good in the case of random delay.This may be because RDCC is a randomized algorithm. For future work other different problems like matrix multiplication and Gaussian elimination task graphs may be considered and observed how it performs in the presence of faults.

\section{REFERENCES}

[1] Anil Kumar Tripathi, P.K. Mishra, Abhishek Mishra,Kamal sheel Mishra, Benchmarking the clustering algorithms for multiprocessor environments using dynamic priority of modules, Elsevier Applied Mathematical Modelling 36 (2012) 6243-6263.

[2] Alexey Lastovetsky, Parallel testing of Distributed Software, Elsevier Information and Software technology Vol 47 (2005) 657-662.

[3] Cyril Briquet, Reproducible testing of Distributed software with middleware virtualization and simulation, ACM (2008).

[4] Giovanni denaro, Andrea polini, Wolfgang Emmerich, Early performance testing of distributed software applications, ACM (2004).

[5] james D. Herbsleb, Audris Mockus, An Empirical study of speed and communication in globally distributed software development, IEEE transactions on software enginering Vol 29 no. 6 (2003) june 481-494.

[6] raul cretta nunes, Ingrid jansch-porto, Modeling communication delays in distributed systems using time series, IEEE transactions (2002) 268-273.

[7] Yizheng yao, Yingxu Wang, A framework for testing distributed software components, IEEE transactions (2005) 1566-1569.

[8] Roger Ferguson, Bogdan Korel, Generating test data for distributed software using the chaining approach, Elsevier Information and software technology Vol 38 (1996) 343353.
[9] Carl K. Chang, Cheng-Chung Song,Rong-Fa Wang, distributed Software Testing with Specification, IEEE 1990.

[10] Y. K. Kwok, I. Ahmad, Benchmarking and comparison of the task graph scheduling algorithms, Journal of Parallel and Distributed Computing 59 (1999) 381-422.

[11] Kequin Li, Scheduling parallel tasks on multiprocessor computers with efficient power management, IEEE transactions (2010) 978-1-4244-6534, New York, USA.

[12] john A. Stankovic, K. Ramamritham, S.Cheng, Evaluation of a Flexible task scheduling algorithm for distributed hard real time systems, IEEE Transactions on Computers Vol c34 , no. 12 (1985) 1130-1143.

[13] V.S. Tondre, V.M.Thakare, S.S.Sherekar, R.V. Dharaskar, Technical computation and communication delay in distributed system, NCICT (2011) IJCA.

[14] R.C.Nunes,I.J. Porto, Modeling communication delays in distributed systems using time series, IEEE transactions (2002) 1060-9857/02, Brazil.

[15] P.K. Mishra, K.S. Mishra, A. Mishra, A clustering heuristic for multiprocessor environments using computation and communication loads of modules, International Journal of Computer Science \& Information Technology, 2(5):170$182,2010$.

[16] T.H. Cormen, C.E. Leiserson, R.L. Rivest, C. Stein, Introduction to Algorithms, 2nd Edition, MIT Press, 2001.

[17] E. Horowitz, S. Sahni, Fundamentals of Computer Algorithms, W. H. Freeman and Co., 1978.

[18] Y. Langsam, M.J. Augenstein, A.M. Tenenbaum, Data Structures Using $\mathrm{C}$ and $\mathrm{C}++$, 2nd edition, Prentice Hall, 1996.

\section{AUTHOR'S PROFILE}

Anil Kumar Tripathi is Professor of Computer Engineering at Indian Institute of Technology (Banaras Hindu University), Varanasi, India. He received his Ph.D. degree in Computer Science from the same institute; and M.Sc. Engg. (Computer) degree from Odessa National Polytechnic University, Ukraine. His research interests include parallel and distributing computing, and software engineering.He has to his credit more than 50 research papers in International journals. He has co-authored two research monographs: one from Springer USA and other from John Wiley USA. Fourteen students have completed their Ph.D under his supervision.

Kamal Sheel Mishra is M.Tech (Computer Engg.) and working as Associate Professor and Head,Computer Science department in the School of Management Sciences, Varanasi, India. He is having more than 17 years of teaching experience. His research interests include Software Engineering, parallel and Distributed Computing.Currently he is Pursuing Ph.D. from Department of Computer Engineering, Indian Institute of Technology (Banaras Hindu University), varanasi,India. 